\title{
A NOVEL GERMLINE TP53 MUTATION IN A PATIENT WITH LI-FRAUMENI SYNDROME - RESOLVING A VARIANT OF UNCERTAIN SIGNIFICANCE
}

\author{
David Douglass ${ }^{1}$, Kimo Stine ${ }^{1}$, and Jason Farrar ${ }^{1}$ \\ ${ }^{1}$ University of Arkansas for Medical Sciences
}

July 24, 2020

\begin{abstract}
Increasing availability of genomic testing poses new challenges to clinicians, particularly where variant interpretation from commercial sources may be equivocal. We report a patient with recurrent rhabdomyosarcoma and subsequent bilateral breast cancer who was found to harbor a previously undescribed germline TP53 sequence alteration annotated by the commercial laboratory as a variant of uncertain significance (VUS). By investigating publically available databases of aggregated normal germline and malignant somatic genomic sequences, we conclude that this missense variant, c.476C $>\mathrm{T}$ (p.A159V), is a novel, pathogenic Li-Fraumeni syndrome mutation, and illustrate the utility of these resources in clinical pediatric hematology and oncology practice.
\end{abstract}

\section{A NOVEL GERMLINE TP53 MUTATION IN A PATIENT WITH LI-FRAUMENI SYN- DROME - RESOLVING A VARIANT OF UNCERTAIN SIGNIFICANCE*}

David P. Douglass, M.D., Kimo C. Stine, M.D., Jason E. Farrar, M.D.

Arkansas Children's Research Institute and Department of Pediatrics, University of Arkansas for Medical Sciences, Little Rock, AR

Corresponding author:

David P. Douglass, M.D.

Arkansas Children's Hospital,

1 Children's Way, Slot 512-10,

Little Rock, AR 72202-3591.

Phone: 501-364-1494 Fax: 501-364-4332 dpdouglass@uams.edu

Word Count: Abstract: 99 Main text: 1195

Tables: 0 Figures: 1 Supporting Information files: 0

Abstract: Increasing availability of genomic testing poses new challenges to clinicians, particularly where variant interpretation from commercial sources may be equivocal. We report a patient with recurrent rhabdomyosarcoma and subsequent bilateral breast cancer who was found to harbor a previously undescribed germline TP53 sequence alteration annotated by the commercial laboratory as a variant of uncertain significance (VUS). By investigating publically available databases of aggregated normal germline and malignant somatic genomic sequences, we conclude that this missense variant, c.476C $>$ T (p.A159V), is a novel, 
pathogenic Li-Fraumeni syndrome mutation, and illustrate the utility of these resources in clinical pediatric hematology and oncology practice.

Running Title: A Novel TP53 Mutation in Li-Fraumeni syndrome

Keywords: Li-Fraumeni syndrome, LFS, IARC, TP53, p53, variant of uncertain significance

Abbreviations:

\begin{tabular}{ll}
\hline VUS & Variant of uncertain significance \\
\hline LFS & Li-Fraumeni syndrome \\
DCIS & Ductal carcinoma in situ \\
IARC & International Agency for Research on Cancer \\
COSMIC & Catalogue of Somatic Mutations in Cancer \\
gnomAD & Genome Aggregation Database \\
\hline
\end{tabular}

* Presented as an abstract/poster entitled "A Novel Germline TP53 Mutation in a Patient with Li-Fraumeni syndrome" at the American Society of Pediatric Hematology/Oncology national meeting in New Orleans, May 1-4, 2019.

\section{Introduction:}

Li-Fraumeni syndrome (LFS), an autosomal dominant cancer predisposition syndrome resulting from germline alterations in the tumor suppressor gene TP53, is characterized by early and/or multiple malignancies in multiple family members. First described by Frederick Li and Joseph F. Fraumeni, Jr. in 1969, their seminal paper described four families with multiple cases of early onset malignancies, in particular, soft tissue sarcomas and breast cancer. ${ }^{1,2}$ From this early work and a 12-year prospective study of these four families, a "classic" definition of LFS was developed by 1988: a proband with sarcoma prior to age 45 who has a $1^{\text {st }}$ degree relative with any cancer before age 45 , plus another $1^{\text {st }}$ or $2^{\text {nd }}$ degree relative with any cancer before 45 or a sarcoma at any age. ${ }^{3,4}$ The term "Li-Fraumeni Syndrome" was first used in $1982 .{ }^{5}$ However, it wasn't until 1990 that germline mutations in the TP53 gene were implicated by Malkin and colleagues. ${ }^{6}$ Chompret et al. revised the classic definition in 2001 in order to optimize sensitivity and specificity. ${ }^{7}$ Most recently, Bougeard et al. refined the Chompret definition in $2015,{ }^{8}$ recommending patients meeting at least one of these criteria should be screened for TP53 alterations:

1. Familial presentation: Proband with LFS spectrum tumor (premenopausal breast cancer, soft tissue sarcoma, osteosarcoma, CNS tumor, or adrenocortical carcinoma) before age 46 AND at least one $1^{\text {st }}$ or $2^{\text {nd }}$ degree relative with LFS spectrum tumor (except breast cancer if proband has breast cancer) before age 56 or multiple tumors.

2. Multiple primitive tumors : Proband with multiple tumors (except multiple breast tumors), 2 of which belong to LFS tumor spectrum and first of which occurred before age 46.

3. Rare tumors : Proband with adrenocortical carcinoma, choroid plexus tumor, or embryonal anaplastic rhabdomyosarcoma regardless of family history.

4. Early-onset breast cancer : Before age 31 regardless of family history. We pursued germline TP53 analysis of a 17-year-old female with bilateral breast cancer following recurrent rhabdomyosarcoma who also had more than one family member with early breast cancer. Sequencing revealed a germline missense mutation c.476C > T (NM_000546.5; p.A159V) that was annotated by the commercial sequencing lab as a VUS. This is the first report of this TP53germline mutation in LFS and illustrates utilizing publically available aggregated sequencing data to determine if a VUS may actually be pathogenic.

\section{Case Description:}

An African-American female presented at 2 years of age with localized alveolar rhabdomyosarcoma of the left shoulder which was treated with vincristine, actinomycin D, cyclophosphamide, topotecan and radia- 
tion (36 Gy). She was disease-free until age 11 when she developed a local recurrence within the radiation field that was initially treated with vinorelbine, cyclophosphamide, and temsirolimus. Due to disease progression during therapy she underwent a forequarter amputation. Histopathology showed de-differentiated rhabdomyosarcoma with diffuse anaplasia which was $95 \%$ viable. She received adjuvant chemotherapy with vincristine, irinotecan and temozolomide and was disease-free at the end of therapy. At age 17, biopsy investigating a right breast mass demonstrated phyllodes tumor. Breast MRI revealed an additional mass in the contralateral breast. Further metastatic work up was negative. She underwent double mastectomy, with pathology demonstrating phyllodes tumor and ductal carcinoma in situ (DCIS) in the right breast and grade 3 invasive ductal carcinoma and DCIS in the left breast. She received adjuvant chemotherapy with 12 weekly doses of paclitaxel followed by 4 cycles of doxorubicin and cyclophosphamide. Two years following completion of therapy, she is 20 years old and remains cancer-free. Her family history included a significant cancer burden, with her paternal great-grandmother, paternal grandmother, and maternal greatgrandmother having breast cancer in their 30's, 40's, and 50's respectively. Neither parent had a history of cancer.

Since she met multiple 2015 Chompret/Bougeard criteria, germlineTP53 sequencing was obtained (Invitae Corp, San Francisco, CA). Analysis showed a heterozygous missense mutation in exon 5 (c.476C>T), resulting in a substitution of valine for alanine at codon 159 (p.A159V). The finding was interpreted by the commercial laboratory as a VUS since an identical missense germline mutation has not been reported in LFS.

Publically available germline genome aggregation databases were queried to confirm the novelty of this mutation. The Genome Aggregation Database (gnomAD, v2.1.1), ${ }^{9}$ yielded no matching germline mutation among over 250 thousand sequenced alleles, demonstrating the extreme rarity of this variant. In addition, the authoritative repository for TP53 mutations, the International Agency for Research on Cancer's (IARC) TP53 Database (Release 20, July 2019),${ }^{10}$ was queried and yielded no matching germline mutation, although two cases in the database showed alterations at residue 159 (Figure 1 ), from alanine (small non-polar) to aspartate (acidic).

Next, aggregated databases of tumor genomic sequences were queried. There were 55 reports of this missense mutation in the IARC TP53tumor cohort, associated most frequently with lung, breast, gastric, ovarian, and CNS tumors. Similarly, query of the Catalogue of Somatic Mutations in Cancer database (COSMIC, v87), ${ }^{11}$ yielded 64 cases including lung, breast, colon, skin, and genitourinary tract tumors, along with 69 additional cases with alternative amino acid alterations at this residue $(43 \mathrm{~A}>\mathrm{P}, 11 \mathrm{~A}>\mathrm{D}, 9 \mathrm{~A}>\mathrm{T}$, and $6 \mathrm{~A}>\mathrm{S}$, Figure 1 ). Additionally, published data on the A159V variant in both human cell culture and yeast models show aberrant transactivation potential and loss of growth suppression resulting from this alteration. ${ }^{12,13}$ In aggregate, these data strongly support the interpretation of this as the pathogenic variant underlying LFS in this patient.

\section{Discussion:}

Since its central role in LFS was described, TP53 has become one of the most studied genes in the scientific literature. Its protein product, p53, acts as a tumor suppressor by regulating wide ranging cellular functions. ${ }^{14}$ As many as $50 \%$ of cancers harbor defects in TP53. ${ }^{10}$ Over 530 distinct sequence alterations in TP53 have been linked to LFS in the IARC database. However, despite decades of extensive genetic investigation, clinical TP53 sequencing may still yield results interpreted as a VUS.

With increased availability and reliance on genomic sequencing in pediatric oncology, the finding of a VUS is likely a familiar phenomenon to most practitioners. In contrast to many loss-of-function mutations such as nonsense mutations, where any variant in the class is presumed to have a common effect (and thus easily annotated), the interpretation of missense variants is less straight forward. In a clinical setting, pathologic attribution generally requires prior observation of the specific codon-level change in a disease-appropriate context. Though it may not affect treatment directly, clarification of a VUS may have important medical or financial consequences to patients and their families in delayed or missed diagnoses, extra testing, and/or 
failure to direct appropriate family studies.

Widespread sequencing studies in healthy populations and in those with cancer can provide important adjunctive data for estimating the likelihood that a variant is pathogenic. With over 140 thousand genome or exome sequences, mostly from families without cancer, gnomAD provides an unparalleled resource to estimate the rarity of individual germline mutations. Similarly, aggregated somatic cancer mutations in COSMIC allow for determination of hotspot mutations and the frequency of observed variants within and among tumor types. IARC's TP53Database offers similar germline and somatic analyses but focuses exclusively on TP53. Although these resources will not resolve every reported VUS, they represent an important addition to the pediatric hematology and oncology toolkit with which anyone interpreting genetic testing should be familiar.

Conflict of Interest statement: None of the authors have relevant conflicts of interest.

\section{References:}

1. Li FP, Fraumeni JF, Jr. Rhabdomyosarcoma in children: epidemiologic study and identification of a familial cancer syndrome. J Natl Cancer Inst. 1969;43(6):1365-1373.

2. Li FP, Fraumeni JF, Jr. Soft-tissue sarcomas, breast cancer, and other neoplasms. A familial syndrome? Ann Intern Med.1969;71(4):747-752.

3. Li FP, Fraumeni JF, Jr., Mulvihill JJ, et al. A cancer family syndrome in twenty-four kindreds. Cancer research.1988;48(18):5358-5362.

4. Kratz CP, Achatz MI, Brugières L, et al. Cancer Screening Recommendations for Individuals with LiFraumeni Syndrome. Clinical Cancer Research. 2017;23(11):e38-e45.

5. Pearson AD, Craft AW, Ratcliffe JM, Birch JM, Morris-Jones P, Roberts DF. Two families with the Li-Fraumeni cancer family syndrome. J Med Genet. 1982;19(5):362-365.

6. Malkin D, Li FP, Strong LC, et al. Germ line p53 mutations in a familial syndrome of breast cancer, sarcomas, and other neoplasms.Science (New York, NY). 1990;250(4985):1233-1238.

7. Chompret A, Abel A, Stoppa-Lyonnet D, et al. Sensitivity and predictive value of criteria for p53 germline mutation screening.J Med Genet. 2001;38(1):43-47.

8. Bougeard G, Renaux-Petel M, Flaman JM, et al. Revisiting Li-Fraumeni Syndrome From TP53 Mutation Carriers. J Clin Oncol.2015;33(21):2345-2352.

9. Karczewski KJ, Francioli LC, Tiao G, et al. The mutational constraint spectrum quantified from variation in 141,456 humans. Nature.2020;581(7809):434-443.

10. Bouaoun L, Sonkin D, Ardin M, et al. TP53 Variations in Human Cancers: New Lessons from the IARC TP53 Database and Genomics Data.Hum Mutat. 2016;37(9):865-876.

11. Tate JG, Bamford S, Jubb HC, et al. COSMIC: the Catalogue Of Somatic Mutations In Cancer. Nucleic Acids Res. 2019;47(D1):D941-D947.

12. Slovackova J, Grochova D, Navratilova J, Smarda J, Smardova J. Transactivation by temperaturedependent p53 mutants in yeast and human cells. Cell Cycle. 2010;9(11):2141-2148.

13. Kotler E, Shani O, Goldfeld G, et al. A Systematic p53 Mutation Library Links Differential Functional Impact to Cancer Mutation Pattern and Evolutionary Conservation. Mol Cell. 2018;71(1):178-190 e178.

14. Sabapathy K, Lane DP. Therapeutic targeting of p53: all mutants are equal, but some mutants are more equal than others. Nature Reviews Clinical Oncology. 2018;15(1):13-30.

15. Zhou X, Edmonson MN, Wilkinson MR, et al. Exploring genomic alteration in pediatric cancer using ProteinPaint. Nat Genet.2016;48(1):4-6. 


\section{Figure Legends}

Figure 1. A schematic view of TP53, with colored lollipops marking missense germline LFS (illustrated above the gene) and somatic mutations in cancer (illustrated below) reported in the IARC germline (r20) and COSMIC (v87) databases, respectively. The location of key p53 domains is illustrated in the figure legend. Upper Panel: The majority of both germline and somatic TP53 mutations cluster within the DNA binding domain, with highly recurrent alterations at residues 175, 248 and 237 in both conditions, and at position 337 in LFS. Lower panel: An exploded view of the affected region illustrates the lack of previously reported LFS A159V mutations but 2 cases with A159D (green). In cancer sequences, the A159V variant (illustrated in red), seen in our patient, is the dominant alteration at this position, representing nearly half of all codon 159 mutations (64/133; other amino acid changes highlighted in green), which constitute approximately $0.5 \%$ of the reported somatic missense TP53 mutations $(133 / 24,890)$. This visualization was adapted from renderings in ProteinPaint (https://pecan.stjude.cloud/proteinpaint). ${ }^{15}$

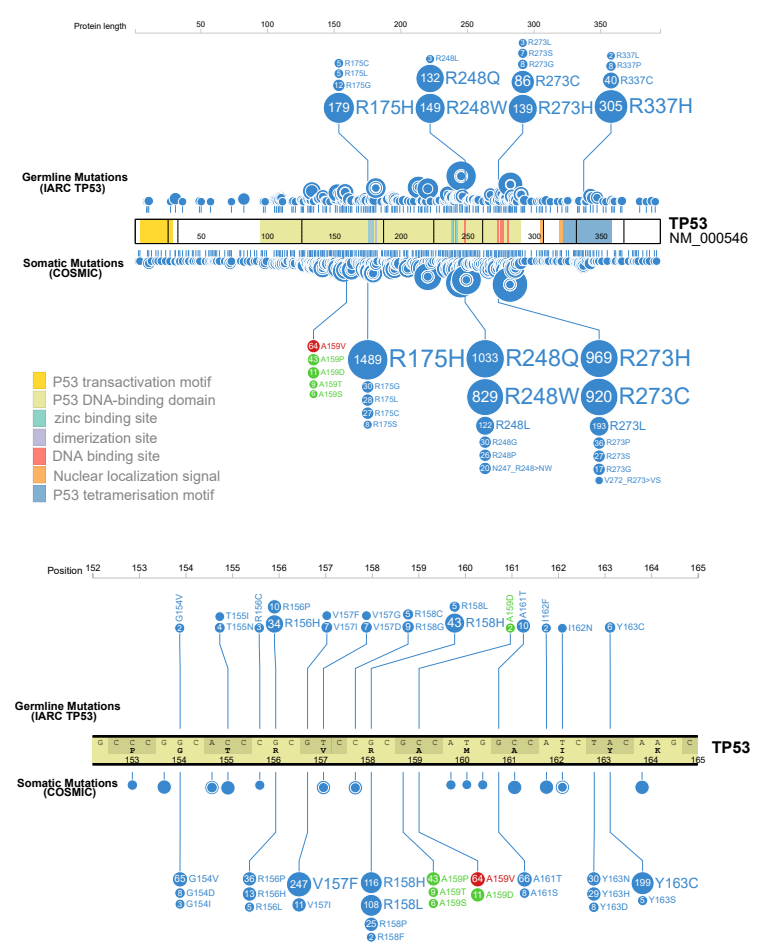

